\title{
Combined umbilical cord blood and bone marrow transplantation from a sibling in a patient with Fanconi anemia
}

\author{
KATARZYNA PAWELEC ${ }^{\prime}$, DARIUSZ BORUCZKOWSKI ${ }^{2}$, TOMASZ OLDAK', MAREK USSOWICZ \\ URSZULA DEMKOW', KRZYSZTOF KAEWAK
}

${ }^{1}$ Department of Pediatric Hematology and Oncology, Warsaw Medical University, Poland

${ }^{2}$ The Polish Stem Cell Bank, Warsaw, Poland

${ }^{3}$ Department of Pediatric Hematology/Oncology and Bone Marrow Transplantation, Wroclaw Medical University, Poland

${ }^{4}$ Department of Laboratory Diagnostics and Clinical Immunology, Medical University of Warsaw, Poland

\begin{abstract}
Fanconi anemia (FA) is an autosomal recessive inherited disorder with progressive bone marrow failure, associated congenital malformation and solid or hematological malignancies. Hematological symptoms usually appear between 4 and 14 years of age, however $10 \%$ of patients are older than 16 years of age at the time of diagnosis. Before the onset of the disease, both the morphology of the peripheral blood and bone marrow smears are within normal range. The first hematologic abnormalities may be thrombocytopenia, leukopenia with granulocytopenia, and anemia. The treatment of choice in children with FA and peripheral blood pancytopenia should be hematopoietic cell transplantation from the best compatible donor. Herein we report on a 12-year-old female patient, the first case in Poland, with $F A$ who received combined cord blood and bone marrow transplantation from a sibling. Due to the risk of late graft rejection, donor lymphocyte infusion (DLI) was carried out twice. No clinical traits of graft $v s$. host disease $(G v H D)$ were observed in the recipient and DLI resulted in a quantitative decrease in mixed chimerism. Now, more than two years after transplantation, the girl remains in a very good condition, with a normal hematopoietic function.
\end{abstract}

Key words: cord blood, bone marrow transplantation, Fanconi anemia.

(Centr Eur J Immunol 2013; 38 (3): 399-402)

\section{Introduction}

Fanconi anemia (FA) is an inherited rare autosomal recessive aplastic anemia associated with developmental anomalies, for the first time described in 1927 [1-3]. The disease more often affects boys (the ratio of boys to girls is 1.2 : 1). Chromosome fragility is pathognomonic for FA. Chromosomes are damaged by ionizing radiations, clastogenic factors such as mitomycin C (MMC) or diepoxybutane (DEB) and hypoxia. Chromosome fragility assessment under the influence of mitogenic factors is a standard test in the diagnosis of FA. Approximately $15 \%$ of patients with FA develop myelodysplastic syndrome (MDS), acute myeloblastic leukemia (AML) and solid tumors, particularly of the gastrointestinal tract, urinary tract, liver, and brain. The "classic" symptoms of FA include: short stature, lack of thumb, microcephaly, skin hyperpigmentations as "cafe-au-lait" spots and a characteristic facial appearance (micrognathia, broad nasal base, epicanthic skin folds). Other physical abnormalities include common birth defects of the circulatory system (patent ductus arteriosus, ventricular septal defect and coarctation of the aorta), urinary tract (kidney failure, ectopic kidney, doubling the urinary tract), gastrointestinal tract (obstruction of the esophagus, duodenum, small intestine, tracheoesophageal fistula - Meckel's diverticulum, umbilical hernia), gonads (sexual glands retardation, impaired spermatogenesis in males) sensory defects (microphthalmia, strabismus, widely spaced eyes, droopy eyelids, deafness, ear abnormalities) [2]. Hematological symptoms usually appear between 4 and 14 years of age,

Correspondence: Katarzyna Pawelec, Department of Pediatric Hematology and Oncology, Warsaw Medical University, Marszalkowska 24, 00-756 Warsaw, Poland, e-mail: katarzyna.pawelec@litewska.edu.pl 
however $10 \%$ of patients are older than 16 years of age at the time of diagnosis. Before the onset of the disease, both the morphology of the peripheral blood and bone marrow smears are within normal range. The first hematologic abnormalities may be thrombocytopenia, leukopenia with granulocytopenia, and anemia. In the full-blown disease, peripheral blood pancytopenia is observed. The treatment of choice for children with FA and significant peripheral blood pancytopenia (neutrophils $<1.0 \times 10^{9} / \mathrm{l}, \mathrm{Hgb}$ level $<8 \mathrm{~g} / \mathrm{dl}$ or platelets $<40-50 \times 109 / 1)$ should be hematopoietic cell transplantation from the best matched donor [1, 2]. In 2006, the fourth report of the European Group for Blood and Marrow Transplantation (EBMT) summarized the clinical management of Fanconi anemia [4, 5]. Hematopoietic stem cell transplantation from an HLA-matched donor is the only treatment with a curative potential [1-3]. Among different options as well umbilical cord blood (CB) stem cells can be transplanted as well. It is always necessary to carry out clinical, hematological and cytogenetic tests to exclude FA in related donors [1-3, 6]. Impaired DNA repair mechanisms in FA result in excessive toxicities after chemotherapy and FA patients must be prepared for transplantation with an appropriate conditioning regimen [7, 8]. Fludarabine (FLU), successfully used for the first time in a child with FA in 1997 by Kapelushnik, is considered as a "gold standard" therapy, but it must be combined with other drugs.

Herein we report the first Polish case of combined matched sibling cord blood and bone marrow transplantation in a child with FA.

\section{Case report}

A twelve-year-old girl was born with duodenal atresia and aortic coarctation with no abnormalities in complete blood cell counts at birth. In the neonatal period, she underwent a corrective surgery of the duodenum, aortic coarctation did not require any surgical procedure. At the age of 4 and 3/12, the patient was diagnosed with isolated thrombocytopenia $\left(45.0-89.0 \times 10^{9} / 1\right)$. In the bone marrow examination, the only abnormality was a decreased number of megakaryocytes. Subsequently in a two-year period, thrombocytopenia progresses and both mild anemia and neutropenia appeared (thrombocytopenia: $18.0 \times 10^{9} / 1$, anemia: Hgb 9.8 g/dl, MCV 107.3 fl, leukopenia: $2.9 \times 109 / 1$ ), bone marrow examination showed a nearly complete absence of megakaryocytes with normal granulopoiesis and erythropoiesis. The patient was diagnosed with idiopathic thrombocytopenia (ITP). Prednisone in a dose of $2 \mathrm{mg} / \mathrm{kg}$ per day was administered for a 6 -week period and the thrombocyte count rose to $35.0-40.0 \times 10^{9} / 1$. At the age of 8 , the patient developed pancytopenia in peripheral blood. Physical examination revealed a very characteristic phenotype: short stature, hyperpigmentation of the skin, "café au lait" spots. Laboratory studies revealed pancytopenia, bone marrow biopsy showed hypocellular mar- row, increased fat space, no malignant cell infiltration, no evidence of malignant disease or myelofibrosis. The diagnosis was confirmed with the use of constitutional karyotype and chromosomal breakage test with mitomycin-C which revealed 46.XX karyotype and an increased number of chromosome breaks $(57 / 71$ metaphases $=80.4 \%)$. At the moment of diagnosis, the patient had no siblings, and HLA search for the unrelated donor was unsuccessful. Peripheral blood parameters gradually declined in the next months. The parents decided to have another child and to collect its umbilical cord blood.

Due to the older sister's disease, the child-to-be-born blood collection was qualified to the "Umbilical Cord Blood Collection for Life Saving" program by the Polish Stem Cell Bank. The processing of collected cord blood (CB) (62 ml) and its cryopreservation was carried out in December 2009. The total number of nucleated cells and CD34+ cells available in the cord blood units were: $76.1 \times 10^{7}$ and $2.7 \times 10^{6}$, respectively. The FA was excluded in a donor with a chromosomal breakage test, and postnatal HLA testing showed full HLA match with the affected sister. The transplant team decided to perform the combined CB and BM transplantation. At age of 11, the patient underwent the transplantation procedure. The conditioning regimen consisted of intravenous busulphan $2 \mathrm{mg} / \mathrm{kg}$ (day -4), fludarabine $5 \times 30 \mathrm{mg} / \mathrm{m}^{2}$ (days from -8 to -4 ), ATG Fresenius $3 \times 20 \mathrm{mg} / \mathrm{kg}$ (days from -3 to -1 ), and OKT-3 (days from +1 to +20 ) (GEFA02 protocol). The transplant material consisted of $\mathrm{CB}$ and $\mathrm{BM}$ stem cells and contained 3.98 million CD34 cells/kg of body weight. Both the donor and the recipient had compatible $\mathrm{O}$ Rh (+) blood group. The patient received GvHD prophylaxis with CSA administered from day +8 . Due to its nephrotoxicity, CSA was replaced with MMF.

The hematologic recovery was stimulated with G-CSF from day +10 to +20 after the transplant, ANC $>0.5 \times 10^{9} / 1$ was reached on day +18 . The first assessment of hematopoietic chimerism on day +25 in peripheral blood mononuclear cells showed $100 \%$ allogeneic chimerism. The patient developed the following complications in the peritransplant period: hemorrhagic cystitis caused by BKV and asymptomatic cytomegalovirus reactivation. In the following months, the mixed hematopoietic chimerism in peripheral blood mononuclear cells was increasing and the immunosuppression was stopped on day +83 . Despite immunosuppression tapering, the trend for increasing mixed chimerism was observed with $24 \%$ of autologous cells in MNC and 68\% in CD3+ compartments. Due to the risk of graft rejection, on day +139 ( 1 million CD3 cells $/ \mathrm{kg}$ of the recipient body weight) and +403 (3 million CD3 cells/kg BW) the donor lymphocyte infusion (DLI) were carried out (Fig. 1). No clinical traits of GvHD were observed in the recipient, however the single infusion of the donor's lymphocytes resulted in a quantitative decrease in mixed chimerism. 


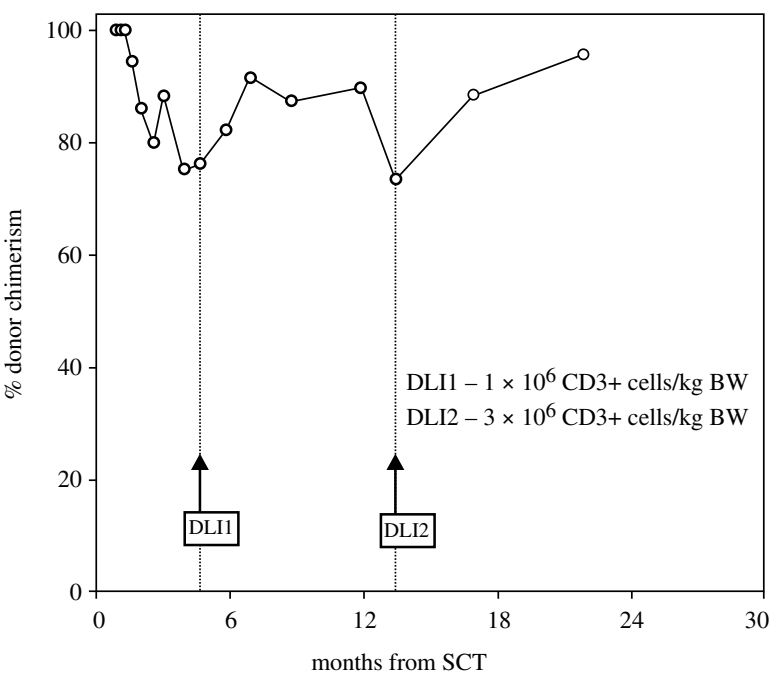

Fig. 1. Donor chimerism after DLI infusion

Currently, more than 2 years after transplantation, the patient remains in a very good condition and exhibits almost full donor chimerism with a normal hematopoietic function.

\section{Discussion}

The first successful transplantation of umbilical cord blood cells from HLA-matched sister was performed in a 5-year-old boy with Fanconi anemia in 1988 [2]. During pregnancy, HLA tests were performed and FA was excluded in his sister. Currently, 24 years after the transplant, the recipient is alive and has normal peripheral blood parameters. Umbilical cord blood has been shown to be a suitable source of hematopoietic stem cells for hematopoietic reconstitution in the course of genetic, metabolic, immunological, hematological and oncological diseases in children [9]. Herein we described a case of FA patients successfully treated with umbilical cord blood transplant enriched with bone marrow stem cells collected from HLA-matched newborn brother. The enrichment with bone marrow hematopoietic stem cells was necessary due to the age and weight disparities between the donor and the recipient. The conditioning toxicities were minor, engraftment was successful and proved the good tolerance and efficacy of GEFA02 protocol [Ebell W., personal communication]. The current guidelines for conditioning of patients with FA mainly include fludarabine as a gold standard, first used in 1997 in a child with FA [8]. ATG/OKT-3 has been used in GEFA02 protocol to avoid both Graft versus Host Disease (GvHD) and graft rejection, which might occur after such a reduced intensity conditioning regimen. GEFA02 protocol includes also low-dose busulphan, as added to the conditioning regimen has resulted in a particularly good outcome in pediatric patients with FA $[7,10]$. In the presented case, hema- tologic recovery was prompt: absolute neutrophil count $>0.5 \times 10^{9} / 1$ was reached after 18 days and was similar to that observed in Maschan's study [7]. The use of ATG may be complicated by infections, as in the described case hemorrhagic cystitis caused by BKV and asymptomatic CMV reactivation were noted. $\mathrm{BK}$ virus-induced infections are frequently observed in patients after hematopoietic cell transplantation as well as the reactivation of herpes viruses [5, 11-13]. The availability of donor blood/bone marrow for posttransplant immunological manipulations played a crucial role in graft rejection prophylaxis and establishing sustained and stable donor chimerism. In the setting of non-malignant disease, GVHD confers no advantage and therefore donor lymphocyte infusion (DLI) with the associated high risk of GVHD, has been only used exceptionally $[14,15]$. Nevertheless, the presented case demonstrates efficacy of DLI in the induction of donor chimerism.

Although UCB are widely performed, this is the first case in Poland of successful cord blood and bone marrow transplantation in a child with FA [16]. The recipient is currently in a good condition and her blood smear remains normal. Her donor is also in good health and developing normally. The awareness of family cord blood banking is a new phenomenon in Poland. The presented case can motivate parents to secure the cord blood material for future therapy of family members.

\section{The authors declare no conflict of interests.}

\section{References}

1. Soulier J (2011): Fanconi anemia. Hematology Am Soc Hematol Educ Program: 492-497.

2. Gluckman E, Broxmeyer HA, Auerbach AD, et al. (1989): Hematopoietic reconstitution in a patient with Fanconi's anemia by means of umbilical-cord blood from an HLAidentical sibling. N Engl J Med 321: 1174-1178.

3. Dalle JH (2008): HSCT for Fanconi anemia in children: factors that influence early and late results. Bone Marrow Transplantation 42: 51-53.

4. Ljungman P, Bregni M, Brune M, et al: European Group for Blood and Marrow Transplantation (2010): Allogeneic and autologous transplantation for haematological diseases, solid tumours and immune disorders: current practice in Europe 2009. Bone Marrow Transplant 45: 219-234.

5. Ljungman P, Hakki M, Boeckh M (2011): Cytomegalovirus in hematopoietic stem cell transplant recipients. Hematol Oncol Clin North Am 1: 151-169.

6. Locatelli F, Zecca M, Pession A, et al.; Italian pediatric group (2007):The outcome of children with Fanconi anemia given hematopoietic stem cell transplantation and the influence of fludarabine in the conditioning regimen: a report from the Italian pediatric group. Haematologica 92: 1381-1388.

7. Maschan AA, Trakhtman PE, Balashov DN, et al. (2004): Fludarabine, low-dose busulfan and antithymocyte globulin as conditioning for Fanconi anemia patients receiving bone mar- 
row transplantation from HLA-compatible related donors. Bone Marrow Transplan 34: 305-307.

8. Kapelushnik R, Or R, Slavin S, Nagler A (1997): A fludarabine-based protocol for bone marrow transplantation in Fanconi's anemia. Bone Marrow Transplant 20: 1109-1111.

9. Cutler C, Ballen KK (2012): Improving outcomes in umbilical cord blood transplantation: State of the art. Blood Rev 26: 241-246.

10. Ljungman P, Urbano-Ispizua A, Cavazzana-Calvo M, et al.; European Group for Blood and Marrow (2006): Allogeneic and autologous transplantation for haematological diseases, solid tumours and immune disorders: definitions and current practice in Europe. Bone Marrow Transplant 37: 439-449.

11. Koskenvuo M, Dumoulin A, Lautenschlager I, et al. (2012): BK polyomavirus-associated hemorrhagic cystitis among pediatric allogeneic bone marrow transplant recipients: Treatment response and evidence for nosocomial transmission. J Clin Virol 12: 1386-6532.

12. Lubin BH, Shearer WT (2007): Cord blood banking for potential future. Transplantation. Pediatrics 119: 165-170.

13. Liao Y, Geyer MB, Yang AJ, Cairo MS (2011): Cord blood transplantation and regenerative potential. Exp Hematol 39: 393-412.

14. Or R, Hadar E, Bitan M, et al. (2006): Safety and efficacy of donor lymphocyte infusions following mismatched stem cell transplantation. Biol Blood Marrow Transplant 12: 1295-1301.

15. Jasinska A, Kalwak K, Trelinska, et al. (2012): Successful haploidentical PBSCT with subsequent T-cell addbacks in a boy with HyperIgM syndrome presenting as severe congenital neutropenia. Pediatr Transplant 17: E37-E40.

16. Smith AR, Wagner JE (2009): Alternative hematopoietic stem cell sources for transplantation: place of umbilical cord blood. Br J Haematol 147: 246-261. 